EDITORIAL. COMUNIDAD, PEDAGOGIA Y SUBJETIVIDAD<smiles>c1ccc(C23CC4CC(CC(C4)C2)C3)cc1</smiles>

EDITORIAL. REVISTA BOLETÍN REDIPE: 9 (7) JULIO 2020 ISSN 2256-1536

\title{
COMUNIDAD, PEDAGOGÍA Y SUBJETIVIDAD
}

\section{COMMUNITY, PEDAGOGY AND SUBJECTIVITY}

\author{
María Fernanda Gil Claros ${ }^{1}$ \\ Grupos de investigación Humanidades y \\ Universidad, Grupo REDIPE
}

\section{RESUMEN}

Comprender el concepto comunidad en la complejidad de las realidades globales implica abrirse a horizontes en los cuales la vida, y específicamente las formas de vivir, están en entredicho por discursos epistemológicos que no han sabido recobrar la fuerza inmanente de la voluntad de vivir en los escenarios vitales, como la comunidad. Es así como la comunidad es repensada en los ámbitos de la vida por el encuentro de subjetividades no propietarias noviolentas $^{2}$ de la existencia, que al exponerse

1 María Fernanda Gil Claros. Colombiana. Doctoranda en filosofía existencial de Maitreya Buddhist University (Argentina). Magister en Estudios Políticos de la Universidad Pontificia Javeriana. Trabajadora Social de la Universidad del Valle. Profesora de tiempo completo de la Universidad Santiago de Cali, Facultad de Comunicación y Publicidad, Departamento de Humanidades. Integrante del grupo de investigación Humanidades y Universidad, grupo de investigación REDIPE maria.gil00@usc.edu.co, mafedarte@gmail.com ORCID: https://orcid.org/0000-0001-7338-6782

2 Se utiliza el término "noviolencia", sin usar dos palabras ni separarlas con guión, para darle un sentido positivo a dicho término, ya que este concepto tiene significado por sí mismo, y no por negación de su contrario: la violen- a un afuera saben proteger la potencia de existir. Es en este contexto que la pedagogía crítica, trasladada a lo experiencial, dispone su lugar en el pensamiento vivo, comprometido con saberes que, como tékhne, construyen lo posible desde las acciones coherentes de una palabra no explicadora.

PALABRAS CLAVE: comunidad, pedagogía crítica, subjetividad, inmunidad.

\section{ABSTRACT}

Understanding the concept of community in the complexity of global realities implies opening to horizons in which life, and specifically the ways of living, are questioned by epistemological discourses that have failed to recover the

cia. Noviolencia es un concepto que acompaña el desarrollo teórico de la educación y la cultura de paz. Montserrat Prieto subraya la necesidad de la palabra "noviolencia" en https:// www.pressenza.com/es/2017/04/la-necesidad-la-palabranoviolencial 
immanent force of the will to live in the vital scenarios, such as the community. This is how the community is rethought in the spheres of life by the encounter of non-possessive nonviolence subjectivities of existence, which when exposed to an outside know how to protect the power to exist.

\section{INTRODUCCIÓN}

El presente artículo da cuenta del proyecto de investigación Pedagogía Crítica constructora de subjetividades comunitarias no violentas, que se encuentra en su fase de exploración documental y cuyo objetivo es realizar un acercamiento al concepto de comunidad, que retorna la vida al momento de relacionarla con ella. Desde lo ético que mira las formas de vivir, el concepto de pedagogía crítica, extendida a la experiencia, se desarrolla desde la palabra como pensamiento vivo.

Como preámbulo, cabe decir que comunidad es un concepto que se ha visto surcado en el trasegar histórico por algunas construcciones teóricas que van desde asumirla como un lugar que otorga tranquilidad, como aquel lugar que confiere seguridad, hasta indagarla bajo ciertas sospechas, por ser un lugar donde es susceptible que se produzca desestabilización de órdenes sociales y políticos; en cambio, otras miradas la aprecian como aquel espacio geográfico estandarizado administrativamente donde es común que las culturas y etnias compartan sus necesidades y problemas sociales.

Es en la modernidad donde el concepto sufre una "disolución", quedando la comunidad sujeta al concepto de sociedad, que a su vez, al menos en el primer momento de constitución de la república, es reducida por la racionalidad contractual moderna en el entramado institucional (Donzelot, 2007). Edificado desde la lógica legal jurídica, la institucionalidad le otorga a la sociedad el carácter de ejercicio racional jurídico, como ser la encargada de formar las uniones y las solidaridades, como regular el vivir junto con los demás, donde lo emocional y/o afectivo tendría poco que decir, ya que termina siendo suplantado por los discursos jurídicos cual ideario constitutivo de la república encarnada en un Estado dador de equidad y bienestar.

Esta configuración moderna trae consigo el diseño piramidal de la razón contractual que tiene como base a la sociedad, como baluarte de la constitución de un orden jurídico que muestra una comunidad reducida a ser la "antesala disuelta" en la sociedad, postulando así una dualidad: la sociedad como el órgano constitutivo del saber jurídico, que fundamentado en el pensamiento moderno antropocéntrico emerge normativamente artificiosa, limpia y/o carente de una cercanía con lo afectivo, con lo emocional, y una comunidad dada desde lo orgánico, desde el vínculo del sentimiento. Como bien lo expresa Tönnies, uno de los clásicos de la exploración de la comunidad, en su libro "Comunidad y Sociedad":

La relación misma, y también la unión, se concibe, bien como vida real y orgánica $-y$ entonces es la esencia de la comunidad-, bien como formación ideal y mecánica -y entonces es el concepto de sociedad.

...Toda vida en conjunto, íntima, interior y exclusiva, deberá ser entendida, a nuestro parecer, como vida en comunidad. La sociedad es lo público, el mundo.

Uno se encuentra en comunidad con los suyos desde el nacimiento, con todos los bienes y males a ello anejo. Se entra en la sociedad como en lo extraño (Tönnies, 1947, p.19).

Es entonces como, en dicha dualidad, se deja ver que el concepto de comunidad se ubica más allá de los postulados de la racionalidad 
moderna, dejando fluir relaciones de cercanías conectadas con lo vivo, como lo son las relaciones de parentesco, de vecindad, de amistad (Tönnies, 1947), que evocan los entramados de subjevidades implicadas con el sentimiento y con el afecto.

En este escenario moderno, de sujeción de la comunidad a la sociedad, se van instalando discursos políticos que evitan o, en el mejor de los casos, reemplazan a la comunidad reconociendo la preponderancia del movimiento obrero, los movimientos sindicales, las organizaciones políticas $o$, en el peor escenario, mediante movimientos nacionalistas y/o dictaduras. De esta forma la comunidad permanece en el ejercicio instrumental de operativizar las direccionalidades bien sea de un movimiento-masa, de un movimiento político o de las políticas de Estado, como sucedió en la década de los 50's cuando la comunidad era vista teóricamente bajo la categoría de organización comunitaria, instrumento geopolítico del mundo colonial para construir estratégicamente territorialidades coloniales, y así poder llegar a lo micro de lo local, atravesando las barreras culturales y de resistencias.

Este fue el papel cumplido por los proyectos, que desligados muchas veces de las propuestas venidas de la gente de a pie, que vivían y padecían el rigor del coloniaje, terminaban instalando bajo la figura del progreso comunidades atrasadas, primitivas, las cuales entregaban su autonomía a cambio de asistencialismo.

En la década de los $60 " \mathrm{~s}$, y específicamente en América Latina, acompañado por los aires de la teoría social crítica, de mayo del 68, del socialismo real y de los acontecimientos vividos bajo las dictaduras militares, emergen pensamientos $y$ propuestas que hacen un llamado hacia el reconocimiento de un pensamiento latinoamericano, un pensamiento de la liberación, donde se le restituyera a la acción su sentido ético, y de la política que operada desde el retorno a los saberes, construye con la gente de a pie gobernabilidades democráticas. Es quizás en este momento en el cual la comunidad, de manera ambigua, avanza hacia la visibilización de lo propio, lo singular de los territorios, de los procesos sociales latinoamericanos con raigambre profundamente colectiva, solidaria y autónoma, donde se reivindican lo cotidiano y lo subjetivo como fuerzas de definición de territorios comunitarios agenciadores de propuestas emancipadoras.

Por consiguiente, y considerando el anterior preámbulo, en momentos de globalización, donde el Estado Nación ha dejado de ser dominante, donde el príncipe ha dejado su trono, es perentorio dar la vuelta $\mathrm{o}$, mejor, dar la cara a la comunidad, para retomar en este mirar los hilos éticos y pedagógicos ligados con formas de vivir dignamente, que permitan pensar comunidades comprometidas con las acciones sociales y políticas, que se repiensen las comunidades que se están instalando o se instalan desde las lógicas de los capitales financieros, como es el caso de aquellas comunidades migratorias que, desplazadas de sus territorios, son sometidas a deterritorializaciones permanentes. Tales desplazamientos traen consigo un cordón humano desnudo frente a las embestidas de los biopoderes, que dejan a su paso comunidades desarraigadas de sus sentidos de vivir, comunidades sin futuro, ofreciendo a cambio comunidades del terror, comunidades de encierro, que dan su libertad a cambio de seguridad (Bauman, 2003).

Es precisamente este panorama el que se configura con el ejercicio del biopoder, productor de comunidades sin mañana, comunidades de clausura $\mathrm{o}$, en el peor desenlace, comunidades mercenarias, que hace necesario mirar de "nuevo" la comunidad, quizás como uno de los caminos para deconstruir discursos de sociedades de control financiero, de 
racionalidades de la gestión del conocimiento. Es posible desde una comunidad pensada más allá de lo exclusivamente institucional, de los espacios exclusivamente políticos administrativos, emprender el camino hacia una comunidad otra que suscite la inquietud de vivir ética y estéticamente junto a los demás, con la angustia existencial de producir biopolíticas para la vida, de regresar a las subjetividades que desde adentro miran el afuera a sabiendas de que en ellas está el afuera, ampliándose el horizonte de las comunidades como espacios de voluntades vitales, que ponen como uno de los sentidos de vivir la pregunta de cómo se está habitando el planeta tierra desde más allá del antropocentrismo.

\section{COMUNIDAD Y VIDA}

En esta reflexión aparece la figura de Roberto Esposito, que al asumir lo que acontece se arriesga a mirar la comunidad desde la biopolítica, invitando a comprender la comunidad más allá de aquel sujeto propietario, más allá de la suma de intersubjetividades, de aquellas subjetividades propietarias o poseedores de cosas. Para ello se emprende el camino de la inmanencia, como el devenir de la potencia de existir, donde se pone en juego la vida, en sus dos formas: la vida biológica, vivir los condicionamientos orgánicos que implica el simple hecho de estar vivo, y el comprometerse con las acciones y los sentidos de crear formas de vivir que cuiden de la vida misma.

Para Espósito hablar de comunidad es mirar la biopolítica como agenciadora de gobernabilidades, lo que implica pensar la política como forma de vida (Espósito, 2006), y de otro lado pensar la inmunidad, como aquel riesgo que se toma en comunidad de exponerse al afuera, que hace que ella retorne al interior, para saberse que lo común no existe como esencia, haciendo así de la comunidad un horizonte, a veces incierto, pero necesario. Es aquí donde dicho pensamiento acompaña a mirar la comunidad como posibilidad de retornar a la inmanencia (potencia-acto), del retorno a las subjetividades no para decir qué hacer sino para construirse en la exposición frente al otro, de retornar con el coraje ético de saberse, desde la voluntad de vivir, agenciador de propuestas en forma de vida, en la cual el dar vendría siendo la disposición de exponerse a ofrecer no a un segundo sino a un tercero que es el posible.

Así, en las comunidades inquietas por la vida se anuncia, se las anuncian como aquellas formas de vivir éticamente junto a los demás, un tejido desde saberes de la gente de a pie, que restituyen al pensamiento su lugar de ser vivo, de ser viviente. Es un pensamiento que recobra una episteme comprometida con lo que acontece en los ámbitos de las subjetividades y las subjetivaciones, del devenir acto, que trasciende la relación epistemológica sujetoobjeto del mundo del afuera, y deja ver los acontecimientos como subjetividades en devenir:

¿Es posible hacer una historia que no tenga por referencia un sistema del sujeto-objeto -una teoría del conocimiento- y que se dirija en cambio a los acontecimientos del saber y al efecto de conocimiento que les sería interior? EI problema consiste en apreciar la posibilidad de una inversión de la configuración tradicional, que sitúa con carácter previo el conocimiento como forma o facultad, y luego los acontecimientos del saber como actos singulares que actualizan esa facultad y en ciertos casos pueden modificar su forma. (Foucault, 2012, p.48).

En otras palabras, la comunidad al girar la mirada hacia la vida, deja de ser aquel lugar tranquilo, para ser comprendida como posibilidad de ser, en donde el dar y la inmunidad actúan como 
operadores del emerger de la diferencia dentro de la comunidad. Examinemos mejor estos dos términos:

El dar, como lo deja dicho Espósito (2003), no es un dar desde el sacrificio o desde el sufrimiento, quien da acepta que se está viviendo con otro, ese otro que es un no igual (se da, pero desde la propiedad), sino aquello que al ofrecer lo que se da es libertario, aquello que huye de la posesión jurídica, se da el interior, que no es el ensimismarse, o la introspección, ni muchos menos el intercambio de interiores, que busquen estándares intersubjetivos. No es entonces una comunidad que se forma como corporación o fusión de individuos para crear un individuo mayor, expresado en términos de líder, pero tampoco es un agregado de intersubjetividad, para formar identidad. Es un dar que libera a la comunidad de aquellas subjetividades cosificadoras y dueñas de los destinos de la gente que, bajo el dispositivo del pacto moral de un jefe, de un líder, se da la seguridad jurídica para tomar la vida de los demás, el destino de los demás, entregando una voluntad débil y obediente a las órdenes, un vacío

La inmunidad es lo contrario de lo común, es el vaciamiento de toda esencia, de todo referente, es como estar de cara desnuda frente a lo inesperado; ese inesperado que es lo negativo de la vida, pero que la contiene, que para dejar emerger la vida se expone a ese riesgo de morir. En palabras de Espósito (2003), lo inmunitario contiene lo comunitario, la communitas, por ello el término inmunidad es el eslabón que le faltaba a la diada modernidad-política. De hecho, la modernidad capitalística ${ }^{3}$ le ha atribuido con la figura contractual un sentido negativo: el ser un sujeto de derecho aquel individuo soberano de libertad.

$3 \quad$ El adjetivo refiere la relación o pertenencia intrínseca del capital a la modernidad, en otras palabras, sin capital no hay modernidad, pero la modernidad puede construirse sin la soberanía del capital.
Es comprender lo inmunitario como aquello que se contrae a un interior que no es propiedad, sino inmanencia, y que después de saberse henchido por el saber del afuera, hace que emerja la voluntad de vivir que protege la vida, que emerja vida en lo posible, pues en lo inmune se protege lo posible, lo incierto, ya que aquello que conoció en el acontecimiento de exponerse, le permite crear futuro posible.

Es como si con el dar en la comunidad, entre más se da, más se es colectivo, restituyéndole a la singularidad el sentido de lo impropio, de la fuerza de conservar lo colectivo en el compartir lo que se vive, y lo que se construye en una existencia cuidadora de la forma de vivir, y con la inmunidad, como aquellas voluntades de vivir, que se atreven a exponerse al afuera para proteger lo posible, ligando a los saberes con acciones éticamente coherentes con formas de vivir en coexistencia.

Estas dos características de la comunidad son dos fuerzas constitutivas de la misma, que permiten comprender su sentido ético de saber cuidar en la comunidad el saber, que es como tékhne, en tanto permite la posibilidad de exponerse al otro, en acontecimientos que ponen en juego no sólo el mero hecho biológico de vivir, sino también el construir formas de vivir, que eviten gobernabilidades destructoras de la vida, y sean potencias de existir.

Por estas razones, el saber en el ámbito de la biopolítica, es un posible, y no algo instituido, donde el otro, los otros, hacen parte del posible cuando se lo considera potencia de saberse ser, cuando se tiene el coraje de exponerse con lo que se hace, a lo incierto, a lo no dicho, dado en el escenario de lo cotidiano. Es entonces en el escenario de lo cotidiano donde acontece la exposición de manera paradójica, ya que de un lado se asiste a los actos de repetición, a los actos de rutina, donde pervive la inercia de saberes predictivos del hacer, y actos de los cuales, en 
el "riesgo" inmunitario, al cuidar de lo impropio, emergen saberes ligados directamente con la incertidumbre de vivir colectivamente gozosos, de los actos éticos de hacer de los saberes las acciones creativas de permitir el vivir (de) lo bello, así éste no sea productivo.

\section{EL LUGAR DE LA PEDAGOGÍA EN LA COMUNIDAD}

De un lado, en los ámbitos de la crítica y en el contexto de la vida en comunidad, la pedagogía es comprender con la escuela de Frankfurt el legado de la crítica en la teoría social crítica, en la cual la subjetividad desde lo filosófico, la psicología profunda y una praxis social comprometida con la emancipación humana, hizo emprender la travesía de lo pedagógico hacia caminos de repensarse lo epistémico, legado que en América Latina es retomado de manera vigorosa por Paulo Freire y la propuesta de educación crítica, donde el conocimiento entra en los ámbitos subjetivos de tejer conciencias, y lo pedagógico trabaja directamente con los mundos de los "dominados", para así acompañar los despertares hacia los cambios sociales. Este cambio a partir crítica se ubica en el pensamiento, es un saber en cuanto práctica de saber pensar lo que se hace, luego aquí lo importante para el pensamiento es la acción misma, como verbo, para trabajar la voluntad de poder.

De otro lado, extender dicho legado en los hilos de la comunidad y la vida, es retornar a los saberes, en el cual los actos superen la dicotomía sujeto-objeto del mundo moderno, y entrar en los acontecimientos del vivir, donde la pedagogía en su perspectiva crítica se encarga o se ocupa de mirar los saberes desde el adentro o desde sus adentros de comunidad, pero no lo hace de cualquier manera, sino de llevar o torcer la crítica hacia los ámbitos de saberes inquietos, de acontecimientos donde emergen las subjetividades, en situaciones afectivas $y$ afectadas por la posibilidad de un tercero.

Es una crítica no de la palabra orientadora, sino cuidadora de la palabra dicha, de la palabra que viene, punzada por el sentir de los acontecimientos, para asumir en las acciones el hecho de vivir y tener el coraje de dar desde una forma de vivir.

...la palabra, simplemente, viene, nos viene.

Por eso, la palabra oída es la palabra "que nos está destinada". No la palabra que nos constituye como destinatarios, es decir, la que se propone hacer alguna cosa con nosotros, ni tampoco la que nosotros buscamos desde nuestras preguntas, 0 desde nuestras inquietudes, desde lo que queremos en definitiva, sino aquella en la que "oímos" nuestro destino. (Larrosa, 2003, p. 44).

Es entonces una pedagogía crítica extendida a la experiencia que, al darse en los encuentros de las subjetividades, teje subjetivaciones, como acciones de la voluntad de vivir, y acoge lo posible que viene con la palabra acto -no la palabra lingüista, sino potencia-acto de experienciar-, deviniendo en lo colectivo de saberse comprometido con la elección-decisión de los instantes acontecidos, en una singularidad que se sabe irreversible. Es entonces una crítica en retorno a lo singular donde la palabra toma sentido ético en lo empírico.

Carácter local de la crítica, lo cual no quiere decir, me parece, empirismo obtuso, ingenio o necio, y tampoco eclecticismo blando, oportunismo, permeabilidad a cualquier empresa teórica, niascetismo un poco voluntario, reducido a la mayor magrura teórica posible. Creo que ese carácter, lo de la crítica, indica en realidad, algo que 
es una especie de producción teórica autónoma, no centralizada, vale decir, que no necesita, para establecer su validez, el visado de un régimen común. (Foucault, 2001, p. 20).

Por consiguiente, una pedagogía extendida a la experiencia hace de la palabra el acto de pensarse aquellos saberes preocupados por la formación de un éthos, colectivo, que se da más allá de los saberes estandarizados de la educación, donde el lugar de lo pedagógico viene dado en el pensamiento. Lo pedagógico es saber pensarse el presente, atravesado por los instantes de los acontecimientos, es saber comprender y decidir que el destino de lo impropio se da más allá de aquellos saberes que como conocimientos predictivos, llenan de contenidos lo formativo, para dar paso a las expresiones explicativas, instituidas en aquel capacitador o profesor limitado al ejercicio de profesar lo que ya está escrito, como bien lo dijera Rancière (2003) en su crítica dirigida al maestro explicador:

Explicar alguna cosa a alguien, es primero demostrarle que no puede comprenderla por sí mismo. Antes de ser el acto del pedagogo, la explicación es el mito de la pedagogía, la parábola de un mundo dividido en espíritus sabios y espíritus ignorantes, espíritus maduros e inmaduros, capaces e incapaces, inteligentes y estúpidos. (Rancière, 2003, p. 8).

Entonces, es ir a la cacería de aquellos saberes circundados en lo cotidiano, donde el contenido de los conocimientos es dado desde los pensamientos vivos, que hacen que la experiencia llene de contenido a los conocimientos, manteniéndolos siempre en el borde. Tal exposición que lo que son es para comprender que no son absolutos, ni únicos:
Cuando hablamos de pensamiento, ¿a qué nos referimos? A un pensamiento que se entiende como una postura, como una actitud que cada persona es capaz de construirse a sí misma frente a las circunstancias que quiere conocer. No se trata de decir: tengo los conceptos y construyo un discurso cerrado, lleno de significaciones; se trata más bien de partir de la duda previa, anterior a ese discurso cerrado, y formularse la pregunta ¿cómo me puedo colocar yo frente a aquello que quiero conocer? (Zemelman, 2009, p. 1).

La pedagogía crítica se ocupa de cuidar ese carácter, que se dispone a pensarse lo vivo con su voluntad de vivir, en contextos donde los saberes, por el contrario, son devenires expuestos a saberes instituidos que, como explicadores, desean controlar las voluntades de saberse dar un lugar no lugar en las singularidades de su existencia.

Esto hace que la pedagogía entable con la educación una relación de borde donde el pensamiento se expone, como algo vivo, a los rigores de los conocimientos, y al saberse expuesto se inmuniza frente a lógicas predictivas y discurso estandarizados.

Así, el pensamiento ronda a la educación, desde la acción, que en la palabra es verbo, siendo la conjunción del disponerse voluntario y la decisión de la elección, en el cual el conocimiento recobre el sentido de ser aquel viaje que se emprende, sin ninguna pretensión de dominio o control, haciéndolo el trasegar de instantes a los cuales se decide entrar para salir otro, eso otro diferente, ese otro posible de hacer del pensar el acto vital de vivir. 
EDITORIAL. COMUNIDAD, PEDAGOGIA Y SUBJETIVIDAD $[1$

\section{CONCLUSIÓN}

En momentos de globalización, donde la lógica impuesta es la devastación de los mercados financieros, y la imposición del sin futuro, y donde la vida en sí misma está ocupando el centro de atención por lógicas de gobernabilidad que en la pretensión de controlarla, ponen en extensión sus potencias de existir, se hace necesario retornar a la comunidad, pero en relación a la vida.

En este contexto es que la comunidad se liga al tema de la biopolítica, si se la comprender como la construcción de gobernabilidades que potencia la vida, donde la subjetivaciones como cruces, encuentros y desencuentros de la subjetividades libertarias, dan paso a la otredad como posible, y no como instituido. Es decir, la comunidad cuida del otro, pero no del que necesita, sino de aquel posible que aún no es, pero que existe siendo.

Este es el lugar no lugar de la pedagogía crítica, que al ligarse con la vida deviene experiencia, en la cual el pensamiento es la acción ética, experiencia que hace que el pensamiento, al exponerse al afuera del conocimiento predictivo, haga de la palabra tanto en el aula como por fuera de ella, las acciones que apunta en lo cotidiano, el potencializar aquella expresión estética de los saberes que subsisten en ella.

Por tanto, la pedagogía de la experiencia al fusionarse a la vida, retorna a la cotidianidad, pero no ya para ser mirada negativamente, como aquel lugar donde suceden los saberes cual doxas equivocadas, sometidas a lo material, a las cogniciones capitalísticas, sino cual saberes que como tékhne, innovan y fabrican con la fuerza de la voluntad de vivir, formas de vivir, formas donde la palabra sea como pensamientos que construyen comunidades cuidadoras de lo posible vital.

\section{REFERENCIAS BIBLIOGRÁFICAS}

Esposito, R. (2003). Communitas. Buenos Aires: Amorrortu.

Esposito, R. (2005). Immunitas. Buenos Aires: Amorrortu.

Esposito, R. (2006). Bíos. Buenos Aires: Amorrortu.

Foucault, M. (2012). Lecciones sobre la voluntad de saber. Buenos Aires: Fondo de Cultura Económica.

Foucault, M. (2002). La hermenéutica del sujeto. México: Fondo de Cultura Económica.

Larrosa, J. (2003). Entre lenguas. Barcelona: Laertes

Larrosa, J. (2003). La experiencia de la lectura Barcelona: Laertes

Rancière, J. (2003). El maestro Ignorante. Barcelona: Laertes.

Zemelman, H. (2009). Pensar teórico y pensar epistémico: los retos de las ciencias sociales latinoamericanas. En: Instituto Pensamiento y Cultura en América A. C. Recuperado hora: 9:20 am, junio 3 de 2019, de: https://repository.unad.edu.co/ bitstream/10596/5564/1/Documento7. pdf 